OPEN ACCESS

Edited by: József Tímár

Semmelweis University, Hungary

*Correspondence:

Fuyou Zhou

ayzhoufuyou@163.com

Received: 18 April 2021 Accepted: 09 June 2021 Published: 09 July 2021

Citation:

Zhang $N$, Liu Y, Yang $H$, Liang $M$, Wang $X$, Wang $M$, Kong J, Yuan X and Zhou $F$ (2021) Clinical Significance of Fusobacterium nucleatum Infection and Regulatory $\mathrm{T}$ Cell Enrichment in Esophageal Squamous Cell Carcinoma.

Pathol. Oncol. Res. 27:1609846. doi: 10.3389/pore.2021.1609846

\section{Clinical Significance of Fusobacterium nucleatum Infection and Regulatory $T$ Cell Enrichment in Esophageal Squamous Cell Carcinoma}

\author{
Ning Zhang ${ }^{1}$, Yiwen Liu $^{2}$, Hong Yang ${ }^{3}$, Mengxia Liang ${ }^{2}$, Xiaopeng Wang ${ }^{1}$, Min Wang ${ }^{1}$, \\ Jinyu Kong ${ }^{2}$, Xiang Yuan ${ }^{2}$ and Fuyou Zhou ${ }^{1 *}$ \\ ${ }^{1}$ Anyang Tumor Hospital, The Fourth Affiliated Hospital of Henan University of Science and Technology, Anyang, China, \\ ${ }^{2}$ Henan Key Laboratory of Cancer Epigenetics, Cancer Institute, The First Affiliated Hospital, College of Clinical Medicine, \\ Henan University of Science and Technology, Luoyang, China, ${ }^{3}$ School of PE, Henan University of Science and Technology, \\ Luoyang, China
}

A variety of pathogenic microorganisms promote tumor occurrence and development through long-term colonization in the body. Fusobacterium nucleatum (F. nucleatum) is abundant in precancerous esophageal lesions and is closely related to the malignant progression of esophageal squamous cell carcinoma (ESCC). The invasion of exogenous microorganisms can reshape the immune microenvironment, make the immune system incapacitated, and assist tumor cells in immune escape. A variety of pathogenic microorganisms induce the recruitment of regulatory $T$ cell (Tregs) to allow tumor cells to escape immune surveillance and provide favorable conditions for their own long-term colonization. Tregs are one of the major obstacles to tumor immunotherapy and have a significant positive correlation with the occurrence and development of many kinds of tumors. Because F. nucleatum can instantly enter cells and colonize for a long time, we speculated that $F$. nucleatum infection could facilitate the immune escape of tumor cells through enrichment of Tregs and promote the malignant progression of ESCC. In this study, we found a significant concordance between $F$. nucleatum infection and Tregs infiltration. Therefore, we propose the view that chronic infection of $F$. nucleatum may provide favorable conditions for long-term colonization of itself by recruiting Tregs and suppressing the immune response. At the same time, the massive enrichment of Treg may also weaken the immune response and assist in the long-term colonization of $F$. nucleatum. We analyzed the correlation between $F$. nucleatum infection with the clinicopathological characteristics and survival prognosis of the patients. F. nucleatum infection was found to be closely related to sex, smoking, drinking, degree of differentiation, depth of invasion, lymph node metastasis, and clinical stage. The degree of differentiation, depth of infiltration, lymph node metastasis, clinical stage, and F. nucleatum infection are independent risk factors affecting ESCC prognosis. Additionally, the survival rate and median survival time were significantly shortened in the F. nucleatum infection positive group. Therefore, we propose that long-term smoking and alcohol consumption cause poor oral and esophageal environments, thereby significantly increasing the risk of $F$. nucleatum infection. In turn, $F$. nucleatum infection and 
colonization may weaken the antitumor immune response through Treg enrichment and further assist in self-colonization, promoting the malignant progression of ESCC.

Keywords: Esophageal squamous cell carcinoma, Fusobacterium nucleatum, Prognostic value, regulatory T cells, clinical significance

\section{INTRODUCTION}

Esophageal cancer has very high morbidity and mortality rates [1]. There are approximately 500,000 new cases of esophageal cancer worldwide every year, more than half of which occur in China [2]. Squamous cell carcinoma is the most common histological type of esophageal cancer, accounting for more than $95 \%$ of all esophageal cancers. The prognosis of esophageal squamous cell carcinoma (ESCC) is extremely poor. Although traditional surgery, radiotherapy and chemotherapy, targeted therapy, and immunotherapy are constantly used in comprehensive tumor treatment, the 5 years survival rate of patients with advanced disease is still less than $20 \%$ [3]. The etiology of ESCC is not completely clear, and its risk factors include smoking, drinking, diet, chronic infection, immune dysfunction, and genetic susceptibility. Studies have shown that a variety of pathogenic microorganisms can promote the occurrence and development of tumors through long-term colonization in the body [4-6]. Although the mechanism of pathogenic microorganism infection in tumors is not completely clear, the removal of pathogenic microorganisms is helpful to control the malignant progression of tumors.

Fusobacterium nucleatum (F. nucleatum), a conditionally pathogenic bacterium that colonizes in the oral cavity, can cause a microecological imbalance by altering its population [7]. Due to the absence of venous valves in the oral and maxillofacial regions, $F$. nucleatum can easily spread throughout the body in the circulation, participate in the progression of many diseases [8], and is closely related to the occurrence and development of many tumors [9]. Studies have shown that F. nucleatum is abundant in precancerous esophageal lesions [10], and the survival time of patients with ESCC infected with $F$. nucleatum is significantly shortened [11]. Although the specific pathogenic mechanism is not clear, F. nucleatum is closely related to the malignant progression of ESCC. Previous studies on the relationship between F. nucleatum infection and ESCC have mostly focused on tumor cells themselves, but there is little discussion on the role of the tumor microenvironment. In fact, the occurrence and development of tumors is closely related to the tumor microenvironment [12-13]. The tumor microenvironment not only provides a rich material basis for the malignant behavior of the tumor but also regulates immune cells to prevent their normal function, leading to tumor immune escape and promoting malignant progression [14]. Immunosuppression mediated by regulatory $T$ cells (Tregs) is not only one of the important mechanisms leading to the immune escape of tumor cells but also a key obstacle in tumor immunotherapy [15-16]. Tregs, a subset of $\mathrm{CD}^{+} \mathrm{CD} 25^{+} \mathrm{FoxP} 3^{+}$ regulatory $T$ cells with immunosuppressive functions, play an important role in maintaining immune tolerance and regulating the level of the immune response [17] and are positively correlated with the malignant progression of tumors. Clinical data show that pathogenic microorganisms in a variety of tumors can recruit Tregs [18-21]. Therefore, it is hypothesized that F. nucleatum infection and Treg enrichment may assist in immune escape of tumor cells and promote malignant progression of ESCC.

In this study, RNAscope and immunohistochemistry (IHC) were used to detect F. nucleatum infection and Tregs infiltration, respectively, in ESCC tissues and to analyze F. nucleatum infection correlation with the clinicopathological features and survival prognosis of patients to provide new strategies and therapeutic tools for the treatment of ESCC.

\section{MATERIALS AND METHODS}

\section{Study Subjects}

For this study, a total of 246 patients with ESCC from Anyang Tumor Hospital (ATH; Anyang, Henan, China) were enrolled after radical resection. Tumor tissue specimens and corresponding paracancerous tissue specimens (esophageal mucosa more than $5 \mathrm{~cm}$ from the edge of the cancer tissue) were collected from each patient for formalin fixation and paraffin embedding. The inclusion criteria were as follows: 1) patients did not receive radiotherapy, chemotherapy, or immunotherapy before the operation; 2) no antibiotics were used within four weeks prior to operation; 3) specimens were taken after therapeutic resection of ESCC; 4) postoperative pathological diagnosis of ESCC was performed; 5) comprehensive case information was provided; and 6) the follow-up period was 60 months (5 years). The exclusion criteria were as follows: 1) preoperative radiotherapy, chemotherapy, or immunotherapy; 2) postoperative pathological diagnosis that was not ESCC or complicated with other tumors; 3 ) received immunotherapy after the operation; 4) incomplete case information; and 5) death not caused by ESCC.

The institutional review boards of $\mathrm{ATH}$ reviewed and approved this study (No. 2020-05-B005), and all participants provided written informed consent. Patient information was anonymized and deidentified prior to analysis. Basic patient information, including age, sex, and tobacco and alcohol histories (Smokers were defined as those who smoked at least one cigarette per day and smoked regularly for at least 6 months continuously or cumulatively and were considered smoking positive; others were considered "never smokers" and were considered smoking negative [22]. Alcohol drinkers were defined as those who consumed at least $25 \mathrm{~g}$ of alcohol per day on average and were considered positive for alcohol 
consumption; others were considered "occasional drinkers or never drinkers" and were considered negative for alcohol consumption [23].), and clinical information, including the histological type, degree of tumor invasion and differentiation, lymph node metastasis, tumor-node-metastasis (TNM) stage, and clinical stage, were collected from hospital records. The clinical stage and histological type were based on the 2017 Union for International Cancer Control (UICC)/American Joint Committee on Cancer (AJCC) TNM classification system (eighth edition) for esophageal cancer. ATH has a standard follow-up center, and the patients or their families were contacted every 3 months until the end of follow-up. The end of follow-up was postoperative death, and the surviving patients were followed up to 60 months after the operation. If patients could not be contacted by telephone, they were visited at their homes in the countryside or were instructed to go to the local police station for confirmation.

\section{RNAscope and Scoring}

The RNAscope ${ }^{\circledR} 2.5$ High Definition (HD)-Red Assay is based on Advanced Cell Diagnostics, Inc. (ACD)'s patented signal amplification and background suppression technology. The assay uses a novel and proprietary method of in situ hybridization (ISH) to visualize single RNA molecules per cell in a multitude of sample types mounted on slides. The RNAscope $^{\circledR} 2.5$ HD (ACD) was used in accordance with the manufacturer's instructions [22], with minor modifications (see below). The RNAscope assay was performed on formalin-fixed paraffin-embedded (FFPE) sections using the RNAscope $2.5 \mathrm{HD}$ Assay-RED (322,350, ACD, United States). The paraffinembedded specimens of ESCC tissues and corresponding paracancerous tissues from each patient were sectioned continuously at $3 \mu \mathrm{m}$. The specimens were immediately placed into xylene for dewaxing at $60^{\circ} \mathrm{C}$ for $1.5 \mathrm{~h}(2 \times 5 \mathrm{~min})$ and subjected to ethanol dehydration $(2 \times 1 \mathrm{~min})$. RNAscope hydrogen peroxide $(322,335$, ACD, United States) was added and incubated for $10 \mathrm{~min}$, the slides were placed in boiling $\left(98-102^{\circ} \mathrm{C}\right)$ RNAscope target repair reagent $(322,000$, ACD, United States) for $15 \mathrm{~min}$ and immediately rinsed in distilled water (3 times), and hydrophobic circles were drawn when the slides were completely dry (room temperature). RNAscope Protease Plus (322,331, ACD, United States) was added and incubated for $30 \mathrm{~min}$ at $40^{\circ} \mathrm{C}$ in a HybEZ Hybridization furnace (220 VAC, 310,013, ACD, United States). The slides were then hybridized with an F. nucleatum probe (RNAscope ${ }^{\circledR}$ Probe-B-Fusobacterium-23S-3zz, Cat. No. 486411, ACD, United States) for $2 \mathrm{~h}$ at $40^{\circ} \mathrm{C}$. After hybridization, slides were subjected to signal amplification using the RNAscope ${ }^{\circledR} 2.5 \mathrm{HD}$ Detection Kit-RED (322,360, ACD, United States). The tissue sections that had been incubated with the probe were incubated with Amp 1 (preamplifier) for $30 \mathrm{~min}$ at $40^{\circ} \mathrm{C}$, Amp 2 (background reducer) for $15 \mathrm{~min}$ at $40^{\circ} \mathrm{C}$, Amp 3 (amplifier) at $40^{\circ} \mathrm{C}$, Amp 4 (label probe) for $15 \mathrm{~min}$ at $40^{\circ} \mathrm{C}$, Amp 5 for $30 \mathrm{~min}$ at room temperature, and finally Amp 6 for $15 \mathrm{~min}$ at room temperature. After each of these steps, the sections were rinsed in wash buffer $(310,091$, ACD, United States). Then, the hybridization signal was detected using a mixture of Fast-RED solutions A and B (1:60). After counterstaining with Gill's hematoxylin, slides were dried in a $60^{\circ} \mathrm{C}$ dry oven for $15 \mathrm{~min}$ and sealed with VectaMount (321,584, ACD, United States). The F. nucleatum signal was observed in tumor cells as red granules. As recommended by ACD, a semi-quantitative scoring approach was applied to evaluate the staining results [24]. RNAscope results were examined under a standard bright-field microscope at 20-40× magnification. We used the scoring system provided by the vendor, as follows: 0 : negative, $0-1$ dots/10 tumor cells at $40 \times ; 1+: 1-3$ dots/cell visible at $20-40 \times$ magnification; $2+: 4-10$ dots/cell visible at $20-40 \times$ magnification; $3+:>10$ dots/cell and $<10 \%$ positive cells with dot clusters visible at $20 \times$ magnification; and $4+:>10 \mathrm{dots} /$ cell and $>10 \%$ positive cells with dot clusters visible at $20 \times$ magnification. For each slide, five areas containing the highest number of positive cells or dot clusters were selected. All tumor cells within each field were counted, and then the percentage of positive F. nucleatum mRNA signals was used as the final score. Image acquisition and analysis were performed using 3DHISTECH software (automatic digital slide scanner, Pannoramic ${ }^{\circledR}$ MIDI I, 3DHISTECH, HU) and Lucia G software (Laboratory Imaging, Prague, Czech Republic) for microscopic image analysis [25-26].

\section{IHC and Scoring}

The paraffin-embedded ESCC tissues and corresponding paracancerous tissues from the same patients were fixed with formalin, embedded in paraffin, and serially sectioned at a thickness of $3 \mu \mathrm{m}$. After the wax was dissolved in the oven at $60^{\circ} \mathrm{C}$ for $1.5 \mathrm{~h}$, the samples were immediately placed into xylene for dewaxing ( 3 times in total, $10 \mathrm{~min}$ each time). The samples were successively hydrated in $100 \%, 95 \%, 85 \%$, and $70 \%$ ethanol for $5 \mathrm{~min}$, followed by continuous rinsing with distilled water for $5 \mathrm{~min}$. Antigen repair was performed at $94-98^{\circ} \mathrm{C}$ with citrate buffer solution $(\mathrm{C} 1032$, Solarbio, China) for $15 \mathrm{~min}$ to fully expose antigens. Then, peroxidase blocker was added for $10 \mathrm{~min}$ to reduce endogenous peroxidase activity. After rinsing with phosphate-buffered saline (PBS, P1002, Solarbio, China), goat serum was added to reduce non-specific binding, and background staining was performed for $30 \mathrm{~min}$. Serial sections were incubated with $\mathrm{CD}^{-}, \mathrm{CD} 25^{-}$, and FoxP3-specific primary antibodies (PBS dilution ratio 1:100, Abcam, United Kingdom) overnight at $4^{\circ} \mathrm{C}$. The next day, after the samples were reheated for $1 \mathrm{~h}$ and rinsed with PBS $(3$ times $\times$ $5 \mathrm{~min}$ ), biotin-labelled secondary antibodies were added and incubated at room temperature for $1 \mathrm{~h}$, and the samples were washed with PBS 3 times $\times 5$ min. The SP-9000 SPlink Detection Kit (Biotin-Streptavidin HRP Detection Systems, SP-9000, ZSGB$\mathrm{BIO}$, China) was used according to the manufacturer's instructions. Then, peroxidase-conjugated streptavidin, a biotin-binding protein, was added for sealing at room temperature for $15 \mathrm{~min}$. The samples were washed with PBS ( 3 times $\times 5 \mathrm{~min}$ ) and stained with diaminobenzidine tetrahydrochloride (DAB, DA1010, Solarbio, China) for color detection of antigen-antibody binding. The color was observed under a binocular stereomicroscope (Eclipse 80i, Nikon, Japan), and then the reaction was terminated with distilled water; then, hematoxylin redyeing was performed. The samples were subjected to gradient ethanol dehydration $(70 \%, 85 \%, 95 \%, 100 \%)$ for $1 \mathrm{~min}$ each and then cleared with xylene ( 3 times $\times 3 \mathrm{~min})$. After 


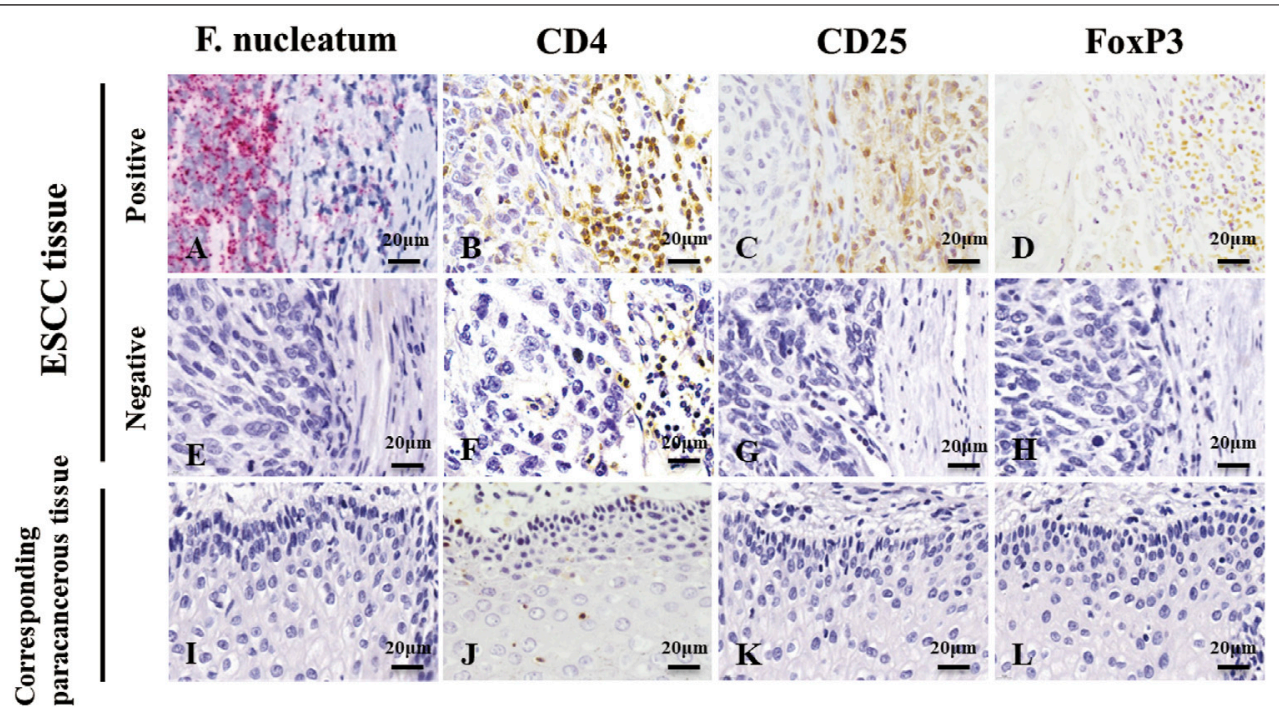

FIGURE 1 |RNAscope detection image and IHC detection image. (A,E,I) are images of $F$. nucleatum in ESCC tissues and corresponding paracancerous tissues, while (B,C,D,F,G,H,J,K,L) are images of Tregs in ESCC tissues and corresponding paracancerous tissues. Red granules can be seen in the cytoplasm of ESCC tissue, indicating F. nucleatum infection (A). In the serial section on the right side, light yellow, tan, or brown coloring was seen on the membrane (CD4 and CD25) or nucleus (FOXP3) of lymphocytes at the same location, indicating infiltration of Tregs (B,C,D). However, most cells were negative in the corresponding paracancerous tissues (I,J,K,L). Original magnification 400x.

air drying, the samples were sealed with neutral gum. The expression of CD4, CD25, and FoxP3 in ESCC tissue and corresponding paracancerous tissue sections was observed by optical microscopy in five randomly selected fields at 400x magnification (Eclipse 80i, Nikon, Japan). The positive sections jointly determined by two senior pathologists were taken as positive controls, and the negative sections (PBS was used instead of the primary antibody) were taken as negative controls. Conflicting results were resolved with a multihead microscope (Eclipse 80i, Nikon, Japan). Samples with light yellow, tan, or brown coloring in the same location of the lymphocyte membrane (CD4 and CD25) or nucleus (FoxP3) in successive sections were considered positive for Tregs infiltration. A semiquantitative scoring scheme based upon the average density of positive-staining cells for the section was utilized for assessment of expression of each immune marker. Five high-power microscopic fields (400 times) in the tissue sections were randomly selected for observation. Each tissue slide was evaluated by two senior pathologists. Staining was scored based on the number of positive-staining cells per 400x field with $1-5$ cells designated as $1+, 6-30$ cells as $2+,>30$ cells as $3+$, and no positive-staining cells as 0 [27]. A final score of $\geq 1$ on all three slides of each patient was defined as positive for Tregs infiltration.

\section{Statistical Analysis}

All statistical analyses were performed with the SPSS statistical package, version 26.0 (SPSS Inc., Chicago, IL, United States): 1) the correlation analysis was performed with the chi-square test, 2) the survival curve was drawn with the Kaplan-Meier method, 3) the difference between survival times was analyzed by the log-rank test, 4) the effect of factors on prognosis was analyzed using Cox regression, and 5) a $p$ value $<0.05$ was considered statistically significant.

\section{RESULTS}

\section{F. nucleatum Infection and Treg Enrichment in ESCC Tissues and Corresponding Paracancerous Tissues}

We used RNAscope and IHC to detect F. nucleatum infection and Treg enrichment in ESCC tissues and corresponding paracancerous tissues (Figure 1) and analyzed the correlation between the two factors. Red granules can be seen in the cytoplasm of ESCC tissues, indicating $F$. nucleatum infection (Figure 1A). In the serial sections, light yellow, tan, or brown coloring was seen on the membrane (CD4 and CD25) or nucleus (FoxP3) of lymphocytes at the same location, indicating the positive infiltration of Tregs (Figure 1C,D). F. nucleatum infection was significantly correlated with Tregs infiltration (Table 1, $p<0.05$ ). However, most cells in the corresponding paracancerous tissues were negative (Figure 1I,J,K,L). F. nucleatum infection in ESCC tissues was significantly higher than that in the corresponding paracancerous tissues $(p<0.05$, Table 2$)$. Tregs infiltration in ESCC tissues was significantly higher than that in the corresponding paracancerous tissues $(p<0.05$, Table 3).

\section{Correlation of $\boldsymbol{F}$. nucleatum Infection With the Clinicopathological Features of Patients With ESCC}

Among the patients with $F$. nucleatum infection (Table 4), there were significantly more males, smokers, and drinkers $(p<0.05)$, and most of the tumor cells in the positive group were 
TABLE 1 | Correlation between F. nucleatum infection and Treg infiltration in ESCC (chi-squared test).

\begin{tabular}{|c|c|c|c|c|c|}
\hline & & \multicolumn{2}{|c|}{ F. nucleatum } & \multirow[t]{2}{*}{$\chi^{2}$} & \multirow[t]{2}{*}{$\boldsymbol{P}$} \\
\hline & & Positive & Negative & & \\
\hline \multirow[t]{2}{*}{ Tregs } & Positive & $75(97.40 \%)$ & $2(2.60 \%)$ & 195.782 & 0.01 \\
\hline & Negative & $10(5.90 \%)$ & $159(94.10 \%)$ & & \\
\hline
\end{tabular}

TABLE 2 | Comparison of the positive rate of F. nucleatum infection in ESCC tissues and corresponding paracancerous tissues in patients with ESCC.

\begin{tabular}{|c|c|c|c|c|c|}
\hline & & \multicolumn{2}{|c|}{ Carcinoma tissues } & \multirow[t]{2}{*}{$x^{2}$} & \multirow[t]{2}{*}{$P$} \\
\hline & & F. nucleatum positive & F. nucleatum negative & & \\
\hline Corresponding paracancerous tissues & $\begin{array}{l}\text { F. nucleatum positive } \\
\text { F. nucleatum negative }\end{array}$ & $\begin{array}{c}8(100 \%) \\
77(32.35 \%)\end{array}$ & $\begin{array}{c}0(0 \%) \\
161(67.65 \%)\end{array}$ & 15.662 & 0.01 \\
\hline
\end{tabular}

TABLE 3 | Comparison of Tregs infiltration between ESCC tissues and corresponding paracancerous tissues in patients with ESCC.

\begin{tabular}{llccc}
\hline & & \multicolumn{2}{c}{ Carcinoma tissues } & $\chi^{2}$ \\
\cline { 3 - 4 } & & Tregs positive & Tregs negative & \\
\hline Corresponding paracancerous tissues & Tregs positive & $7(100 \%)$ & $0(0 \%)$ & 15.662 \\
& Tregs negative & $70(29.29 \%)$ & $169(70.71 \%)$ & 0.01
\end{tabular}

TABLE 4 | Correlation between F. nucleatum infection and clinicopathological characteristics of patients with ESCC.

\begin{tabular}{|c|c|c|c|c|c|}
\hline \multirow[t]{2}{*}{ Factors } & \multirow[t]{2}{*}{$\mathbf{n}$} & \multicolumn{2}{|c|}{ F. nucleatum } & \multirow[t]{2}{*}{$x^{2}$} & \multirow[t]{2}{*}{$P$} \\
\hline & & Positive & Negative & & \\
\hline \multicolumn{6}{|l|}{ Sex } \\
\hline Female & 83 & $12(14.46)$ & $71(85.54)$ & & \\
\hline \multicolumn{6}{|l|}{ Age (years) } \\
\hline$\geq 60$ & 139 & $42(30.22)$ & $97(70.29)$ & 2.658 & 0.103 \\
\hline \multicolumn{6}{|l|}{ Smoking } \\
\hline Positive & 125 & $73(58.40)$ & $52(41.60)$ & 63.908 & 0.001 \\
\hline Negative & 121 & 12(9.92) & 109(90.08) & & \\
\hline \multicolumn{6}{|l|}{ Alcohol } \\
\hline Positive & 119 & $73(61.34)$ & 46(38.66) & 73.165 & 0.001 \\
\hline Negative & 127 & 12(9.45) & 115(90.55) & & \\
\hline \multicolumn{6}{|l|}{ Differentiation type } \\
\hline Poorly differentiated & 52 & 36(69.23) & $16(30.77)$ & 35.063 & 0.001 \\
\hline \multicolumn{6}{|l|}{ Lymph node metastasis } \\
\hline Positive & 107 & $81(75.70)$ & $26(24.30)$ & 141.785 & 0.001 \\
\hline Negative & 139 & $4(2.88)$ & 135(97.12) & & \\
\hline \multicolumn{6}{|l|}{ Clinical stages } \\
\hline |/II & 151 & $4(2.65)$ & $147(97.35)$ & 175.995 & 0.001 \\
\hline III/IV & 95 & $81(85.26)$ & $14(14.74)$ & & \\
\hline
\end{tabular}


TABLE 5 | Mean and median survival times (months) of patients with ESCC with $F$. nucleatum infection.

\begin{tabular}{|c|c|c|c|c|c|c|c|c|c|c|c|}
\hline \multirow[t]{3}{*}{ Group } & & \multicolumn{4}{|c|}{ Mean $^{a}$} & \multicolumn{4}{|c|}{ Median $^{a}$} & \multirow[t]{3}{*}{$\chi^{2}$} & \multirow[t]{3}{*}{$P$} \\
\hline & & \multirow[t]{2}{*}{ Est } & \multirow[t]{2}{*}{ Std. Error } & \multicolumn{2}{|c|}{$95 \%$ confidence interval } & \multirow[t]{2}{*}{ Est } & \multirow[t]{2}{*}{ Std. Error } & \multicolumn{2}{|c|}{$95 \%$ confidence interval } & & \\
\hline & & & & Lower bound & Upper bound & & & Lower bound & Upper bound & & \\
\hline \multirow[t]{3}{*}{ F. nucleatum } & Positive & 27.906 & 1.844 & 24.292 & 31.520 & 24.000 & 2.764 & 18.582 & 55.703 & 32.294 & 0.001 \\
\hline & Negative & 41.839 & 1.455 & 38.987 & 44.690 & 47.000 & 4.440 & 38.297 & 51.970 & & \\
\hline & Overall & 37.024 & 1.221 & 34.631 & 39.418 & 36.000 & 2.091 & 31.901 & 40.099 & & \\
\hline
\end{tabular}

aEstimation was limited to the longest survival time; "Est." and "Std." represent "estimated" and "standard", respectively.
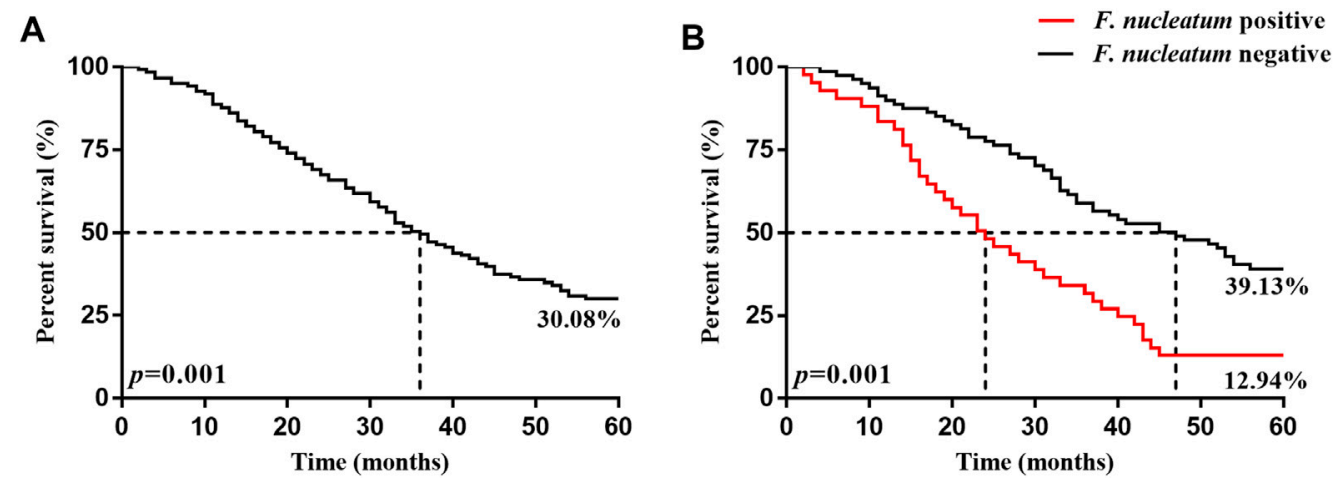

FIGURE 2 | Kaplan-Meier 5 years survival curves of patients with ESCC patients 5 years after surgery. (A) is the 5 years survival curve of patients with ESCC patients. (B) is the 5 years survival curve of $F$. nucleatum-positive and negative patients after surgery.

hypodifferentiated, with deeper tumor tissue infiltration, significantly more lymph node metastases, and more frequent occurrences of clinical stages III/IV $(p<0.05)$.

\section{Correlation of $\boldsymbol{F}$. nucleatum Infection With Survival Prognosis in Patients With ESCC}

The 5 years survival rate and median survival time of the 246 patients with ESCC after surgery were $30.08 \%$ and 36.0 months, respectively. The 5 years survival rate and median survival time in the $F$. nucleatum infection-positive group $(12.94 \%$ and 47.0 months, respectively) were significantly lower $(p<0.05)$ than those in the negative group $(39.13 \%$ and 24.0 months, respectively), as presented in Table 5 and Figure 2.

\section{Cox Regression Analysis of Factors Affecting the Prognosis of Patients With ESCC}

A total of 246 patients with ESCC successfully completed postoperative follow-up, with the death due to ESCC as the endpoint event, and univariate Cox regression analysis was conducted, $F$. nucleatum infection (positive $=1 /$ negative $=0$ ), sex (male $=1 /$ female $=0)$, age $(\geq 60=1 /<60=0)$, smoking (positive $=1 /$ negative $=0$ ), alcohol consumption (positive $=1 /$ negative $=0$ ), degree of tumor differentiation (poor $=1 /$ moderate/high $=0$ ), depth of infiltration ( $\geq$ adventitia $=1 /<$ adventitia $=0)$, lymph node metastasis
( positive $=1 /$ negative $=0)$, and clinical stage $(\mathrm{III} / \mathrm{IV}=1 / \mathrm{I} / \mathrm{II}=0)$ were used as independent variables. The data showed that sex, smoking, alcohol consumption, degree of tumor differentiation, depth of infiltration, lymph node metastasis, clinical stage, and F. nucleatum infection were associated with overall survival $(p<0.05)$. Confounding factors (sex and age) were included, and variables that were significantly associated $(p<0.05)$ with ESCC in the univariate analysis were entered into multivariate Cox regression analysis, which showed that the degree of tumor differentiation, depth of infiltration, lymph node metastasis, clinical stage, and F. nucleatum infection are independent risk factors that affect the prognosis of ESCC $(p<0.05)$, as presented in Table 6.

\section{DISCUSSION}

ESCC has high morbidity and mortality rates and an extremely poor prognosis, and it is difficult to diagnose early. Therefore, it is particularly important to identify accurate early indicators, effective preventive measures, and targeted therapeutic approaches. For a long time, oncological research on chronic infection by pathogenic microorganisms has been lacking. In fact, a variety of pathogenic microorganisms can cause long-term colonization in tumor cells by reshaping the host immune microenvironment, leading to tumor immune escape and promoting malignant progression [28]. For F. nucleatum, one of the most virulent oral pathogenic bacteria [29], its endotoxin 
TABLE 6 | Cox regression analysis of prognostic factors in patients with ESCC.

\begin{tabular}{|c|c|c|c|c|c|}
\hline Clinical variables & B & Wald & $\mathrm{Hr}$ & $95 \% \mathrm{Cl}$ & $P$ \\
\hline \multicolumn{6}{|l|}{ Univariate cox analysis } \\
\hline Sex (male/Female) & 0.743 & 17.888 & 2.103 & $1.490-2.968$ & 0.001 \\
\hline Age $(\geq 60 /<60)$ & 0.030 & 0.038 & 1.030 & $0.763-1.392$ & 0.845 \\
\hline Smoking (positive/Negative) & 1.221 & 54.697 & 3.392 & $2.454-4.689$ & 0.001 \\
\hline Alcohol (positive/Negative) & 1.267 & 60.030 & 3.549 & $2.576-4.890$ & 0.001 \\
\hline Differentiation type (poorly/Moderately-Well) & 0.837 & 22.507 & 2.309 & $1.634-3.263$ & 0.001 \\
\hline Infiltration depth ( $\geq$ Adventitia/ $<$ Adventitia) & 0.982 & 26.969 & 2.670 & $1.843-3.869$ & 0.001 \\
\hline Lymph node metastasis (positive/Negative) & 1.040 & 44.511 & 2.828 & $2.084-3.839$ & 0.001 \\
\hline Clinical stages $(I I I / I V / I / I I)$ & 0.933 & 36.389 & 2.543 & $1.878-3.444$ & 0.001 \\
\hline F. nucleatum (positive/Negative) & 0.856 & 29.868 & 2.353 & $1.731-3.199$ & 0.001 \\
\hline \multicolumn{6}{|l|}{ Multivariate cox analysis } \\
\hline Sex (male/Female) & 0.166 & 0.274 & 1.181 & $0.634-2.199$ & 0.601 \\
\hline Age $(\geq 60 /<60)$ & 0.070 & 0.140 & 1.072 & $0.744-1.546$ & 0.709 \\
\hline Smoking (positive/Negative) & 1.329 & 2.146 & 3.778 & $0.638-22.360$ & 0.143 \\
\hline Alcohol (positive/Negative) & 0.071 & 0.018 & 1.073 & $0.387-2.976$ & 0.892 \\
\hline Differentiation type (poorly/Moderately-Well) & 1.453 & 33.644 & 4.274 & $2.616-6.982$ & 0.001 \\
\hline Infiltration depth ( $\geq$ Adventitia/<Adventitia) & 0.977 & 11.594 & 2.656 & $1.514-4.661$ & 0.001 \\
\hline Lymph node metastasis (positive/Negative) & 1.545 & 15.631 & 3.169 & $1.243-8.084$ & 0.016 \\
\hline Clinical stages (III/IV/I/II) & 1.154 & 5.830 & 4.222 & $1.105-16.134$ & 0.035 \\
\hline F. nucleatum (positive/Negative) & 2.861 & 11.542 & 17.478 & 3.355-91.049 & 0.001 \\
\hline
\end{tabular}

can suppress the body's immune response, thus colonizing the body for a long time and promoting the malignant progression of many tumors, such as oral squamous and esophageal squamous cancers and colon cancer [30].

The mechanism by which the tumor microenvironment is remodeled by pathogenic microorganisms has not been fully understood thus far, but most tumor microenvironments can recruit Tregs to deactivate the body's anti-tumor immunity through intercellular suppression mechanisms as well as through the secretion of immunosuppressive molecules, thus assisting in long-term colonization and the immune escape of tumor cells [31,32]. The number of Tregs in the gastric mucosa of patients with $H$. pylori infection is significantly higher than that in the normal population, leaving the organism in an immunosuppressed state [33] and promoting the malignant proliferation of cancer cells. Cancer cells in patients with hepatitis B virus infection-positive hepatocellular carcinoma can induce cancer cell immune escape by upregulating the TGF- $\beta$ protein and recruiting Tregs in large numbers [34]. Cancer cells in Epstein-Barr virus (EBV) infection-positive Hodgkin's lymphoma patients can recruit Tregs by expressing the chemokine CCL20 via the EBV core antigen EBNA1 [35], which allows cancer cells to evade immune surveillance.

In this study, the positive rates of F. nucleatum infection and Treg enrichment were significantly higher in ESCC tissues than in paracancerous tissues, suggesting that the microenvironment of cancer tissues is more suitable for F. nucleatum colonization and Treg enrichment. Moreover, there was a significant consistency between $F$. nucleatum infection and Tregs infiltration into cancer tissues, suggesting that $F$. nucleatum may provide favorable conditions for long-term colonization by recruiting Tregs and suppressing immune responses. At the same time, the massive enrichment of Tregs could also weaken the immune response and assist in long-term colonization of $F$. nucleatum. Furthermore, we analyzed the correlation of $F$. nucleatum infection with clinicopathological characteristics of patients with ESCC using the chi-squared test and found that $F$. nucleatum infection was significantly correlated with sex, smoking, and alcohol consumption in patients with ESCC. The majority of $F$. nucleatum infection-positive patients were men who smoked and consumed alcohol, which indicated that long-term smoking and alcohol consumption led to a poor oral and esophageal environment, making patients more susceptible to F. nucleatum infection and colonization. The positive rates of F. nucleatum infection was significantly higher in hypodifferentiated ESCC tissues than in tissues with medium-high differentiation, suggesting that $F$. nucleatum infection may correlate with tumor malignancy. Meanwhile, F. nucleatum infection was significantly correlated with the depth of tumor infiltration, lymph node metastasis, and clinical stage, suggesting that F. nucleatum infection may promote the malignant progression of the tumor. This study also found that the degree of tumor differentiation, depth of infiltration, lymph node metastasis, clinical stage, and copositivity for $F$. nucleatum infection were independent risk factors affecting the prognosis of ESCC. The 5 years survival rate and median survival time of patients in the F. nucleatum infection-positive group was significantly lower than those in the respective negative groups, suggesting that effective elimination of $F$. nucleatum may prolong the survival of patients with ESCC. Since tumor development is a multifactorial and multi-step evolutionary process, the specific pathogenic mechanism of $F$. nucleatum needs to be further explored, but disrupting the persistent colonization of $F$. nucleatum in the host and effectively inhibiting the massive enrichment of Tregs are of great importance to actively and 
effectively delaying the malignant progression of ESCC and prolonging the survival of patients.

\section{DATA AVAILABILITY STATEMENT}

The original contributions presented in the study are included in the article/supplementary material, further inquiries can be directed to the corresponding author.

\section{ETHICS STATEMENT}

The studies involving human participants were reviewed and approved by the Institutional Review Board of the Anyang Tumor Hospital. Each study participant signed an informed consent form. The patients/participants provided their written informed consent to participate in this study.

\section{AUTHOR CONTRIBUTIONS}

Conception and design: FZ, NZ; Development of methodology: NZ, YL, XY, JK, and HY; Acquisition of data (acquired and managed patients, provided facilities, etc.): FZ; Analysis and interpretation of data (e.g., statistical analysis, biostatistics, computational analysis): JK, YL, NZ, ML, XW, and MW;

\section{REFERENCES}

1. Sung H, Ferlay J, Siegel RL, Laversanne M, Soerjomataram I, Jemal A, et al. Global Cancer Statistics 2020: GLOBOCAN Estimates of Incidence and Mortality Worldwide for 36 Cancers in 185 Countries. CA A Cancer J Clin (2021) 71:209-49. doi:10.3322/caac.21660

2. Li S, Chen H, Man J, Zhang T, Yin $\mathrm{X}, \mathrm{He} \mathrm{Q}$, et al. Changing Trends in the Disease burden of Esophageal Cancer in China from 1990 to 2017 and its Predicted Level in 25 Years. Cancer Med (2021) 10(5):1889-99. doi:10.1002/ cam4.3775

3. Lagergren J, Smyth E, Cunningham D, and Lagergren P. Oesophageal Cancer. Lancet (2017) 390(10110):2383-96. doi:10.1016/S0140-6736(17)31462-9

4. Carmen Lía. M, Massilva. R, Christian. S, Monique. B, Claire. B, Marine. B, et al. Natural History, Dynamics, and Ecology of Human Papillomaviruses in Genital Infections of Young Women: Protocol of the PAPCLEAR Cohort Study. BMJ Open (2019) 9(6):e025129. doi:10.1136/bmjopen-2018-025129

5. Liu Y, Yuan X, Chen K, Zhou F, Yang H, Yang H, et al. Clinical Significance and Prognostic Value of Porphyromonas Gingivalis Infection in Lung Cancer. Translational Oncol (2021) 14(1):100972. doi:10.1016/j.tranon.2020.100972

6. Li N, Fan X, Wang X, Deng H, Zhang K, Zhang X, et al. PRDM1 Levels Are Associated with Clinical Diseases in Chronic HBV Infection and Survival of Patients with HBV-Related Hepatocellular Carcinoma. Int Immunopharmacology (2019) 73(undefined):156-62. doi:10.1016/ j.intimp.2019.05.012

7. Slade DJ. New Roles for Fusobacterium Nucleatum in Cancer: Target the Bacteria, Host, or Both? Trends Cancer (2021) 7(3):185-7. doi:10.1016/ j.trecan.2020.11.006

8. Han YW. Fusobacterium Nucleatum: a Commensal-Turned Pathogen. Curr Opin Microbiol (2015) 23(undefined):141-7. doi:10.1016/j.mib.2014.11.013

9. Gholizadeh P, Eslami H, and Kafil HS. Carcinogenesis Mechanisms of Fusobacterium Nucleatum. Biomed Pharmacother (2017) 89(undefined): 918-25. doi:10.1016/j.biopha.2017.02.102

10. Baba Y, Iwatsuki M, Yoshida N, Watanabe M, and Baba H. Review of the Gut Microbiome and Esophageal Cancer: Pathogenesis and Potential Clinical
Writing, review, and/or revision of the manuscript: NZ; Administrative, technical, or material support (i.e., reporting or organizing data, constructing databases): NZ, YL, XY, JK, ML, XW, MW, and HY; Study supervision: FZ.

\section{FUNDING}

This work was supported in part by grants from the National Natural Science Foundation of China (81472234 for Shegan Gao), the National Natural Science Foundation of China (81702820 for $\mathrm{XY}$ ), Major project of Science and Technology Department in Henan Province (161100311200 for Shegan Gao), Project of Science and Technology in Henan Province (202102310321 for HY), Project of Science and Technology in Henan Province (202102310129 for JK), Joint Construction Project of Medical Science and Technology Research Program in Henan Province (2018020273 for YL), Project of Science and Technology Plan Medical and Health in Luoyang (1813003A for YL).

\section{CONFLICT OF INTEREST}

The authors declare that the research was conducted in the absence of any commercial or financial relationships that could be construed as a potential conflict of interest.

Implications. Ann Gastroenterol Surg (2017) 1(2):99-104. doi:10.1002/ ags3.12014

11. Yamamura K, Baba Y, Nakagawa S, Mima K, Miyake K, Nakamura K, et al. Human Microbiome Fusobacterium Nucleatum in Esophageal Cancer Tissue Is Associated with Prognosis. Clin Cancer Res (2016) 22(22):5574-81. doi:10.1158/1078-0432.CCR-16-1786

12. Altorki NK, Markowitz GJ, Gao D, Port JL, Saxena A, Stiles B, et al. McGraw Timothy., Mittal Vivek.the Lung Microenvironment: an Important Regulator of Tumour Growth and Metastasis. Nat Rev Cancer (2019) 19(1):9-31. doi:10.1038/s41568-018-0081-9

13. Roumenina LT, Daugan MV, Petitprez F, Sautès-Fridman C, and Fridman WH. Daugan Marie V., Petitprez Florent., Sautès-Fridman Catherine., Fridman Wolf Herman.Context-dependent Roles of Complement in Cancer. Nat Rev Cancer (2019) 19(12):698-715. doi:10.1038/s41568-0190210-0

14. Elinav E, Nowarski R, Thaiss CA, Hu B, Jin C, and Flavell RA. Inflammationinduced Cancer: Crosstalk between Tumours, Immune Cells and Microorganisms. Nat Rev Cancer (2013) 13(11):759-71. doi:10.1038/nrc3611

15. Zhuo C, Xu Y, Ying M, Li Q, Huang L, Li D, et al. FOXP3+ Tregs: Heterogeneous Phenotypes and Conflicting Impacts on Survival Outcomes in Patients with Colorectal Cancer. Immunol Res (2015) 61(3):338-47. doi:10.1007/s12026-014-8616-y

16. Kumar P, Saini S, and Prabhakar BS. Cancer Immunotherapy with Check point Inhibitor Can Cause Autoimmune Adverse Events Due to Loss of Treg Homeostasis. Semin Cancer Biol (2020) 64(undefined):29-35. doi:10.1016/ j.semcancer.2019.01.006

17. Yang L, Wang G, and Xia H. Molecular Mechanism for Impaired Suppressive Function of Tregs in Autoimmune Diseases: A Summary of Cell-intrinsic and Cell-extrinsic Factors. J Cel Mol Med (2020) 24(19):11056-63. doi:10.1111/ jcmm. 15743

18. Lin W, Niu Z, Zhang H, Kong Y, Wang Z, Yang X, et al. Imbalance of Th1/Th2 and Th17/Treg during the Development of Uterine Cervical Cancer. Int J Clin Exp Pathol (2019) 12(9):3604-12.

19. Tang R, Lei Z, Wang X, Qi Q, He J, Liu D, et al. Hepatitis B Envelope Antigen Increases Tregs by Converting CD4+CD25- $\mathrm{T}$ Cells into 
CD4+CD25+Foxp3+ Tregs. Exp Ther Med (2020) 20(4):3679-86. doi:10.3892/etm.2020.9107

20. Ling Z, Shao L, Liu X, Cheng Y, Yan C, Mei Y, et al. Regulatory T Cells and Plasmacytoid Dendritic Cells within the Tumor Microenvironment in Gastric Cancer Are Correlated with Gastric Microbiota Dysbiosis: A Preliminary Study. Front Immunol (2019) 10:533. doi:10.3389/fimmu.2019.00533

21. Urakawa S, Yamasaki M, Makino T, Kurokawa Y, Yamamoto K, Goto K, et al. The Impact of ICOS+ Regulatory T Cells and Helicobacter pylori Infection on the Prognosis of Patients with Gastric and Colorectal Cancer: Potential Prognostic Benefit of Pre-operative Eradication Therapy. Cancer Immunol Immunother (2021) 70(2):443-52. doi:10.1007/s00262-020-02696-4

22. Chinese Preventive Medicine Association. Zhonghua zhong liu Za Zhi [Chinese J oncology] (2021) 43(1):1-7. doi:10.3760/cma.j.cn112152-20210106-00019

23. Wu K, Zhao X, Qi L, Zhou T, Zhang W, Fan Y, et al. A Preliminary Study on the Relationship between Alcohol Consumption and Oral flora in a Natural Population of Middle-Aged and Elderly Men in the Core Region of Southwest China[J]. West China Med (2021) 02:230-7.

24. Wang F, Flanagan J, Su N, Wang L-C, Bui S, Bui A, et al. RNAscope. J Mol Diagn (2012) 14:22-9. doi:10.1016/j.jmoldx.2011.08.002

25. Bullman S, Pedamallu CS, Sicinska E, Clancy TE, Zhang X, Cai D, et al. Analysis of Fusobacteriumpersistence and Antibiotic Response in Colorectal Cancer. Science (2017) 358(6369):1443-8. doi:10.1126/science.aal5240

26. Cioca A, Cimpean AM, Ceausu RA, Tarlui V, Toma A, Marin I, et al. Evaluation of Podoplanin Expression in Hepatocellular Carcinoma Using RNAscope and Immunohistochemistry - A Preliminary Report. Cancer genomics \& proteomics (2017) 14(5):383-7. doi:10.21873/cgp.20048

27. Sparger EE, Murphy BG, Kamal FM, Arzi B, Naydan D, Skouritakis CT, et al. Investigation of Immune Cell Markers in Feline Oral Squamous Cell Carcinoma. Vet Immunol immunopathology (2018) 202:52-62. doi:10.1016/ j.vetimm.2018.06.011

28. Schwabe RF, and Jobin C. The Microbiome and Cancer. Nat Rev Cancer (2013) 13(11):800-12. doi:10.1038/nrc3610
29. Han YW. Fusobacterium Nucleatum: a Commensal-Turned Pathogen. Curr Opin Microbiol (2015) 23:141-7. doi:10.1016/j.mib.2014.11.013

30. Fujiwara N, Kitamura N, Yoshida K, Yamamoto T, Ozaki K, and Kudo Y. Involvement of Fusobacterium Species in Oral Cancer Progression: A Literature Review Including Other Types of Cancer. Ijms (2020) 21(17): 6207. doi:10.3390/ijms21176207

31. Li W-M, and Liu H-R. CCL20-CCR6 Cytokine Network Facilitate TregActivity in Advanced Grades and Metastatic Variants of Hepatocellular Carcinoma. Scand J Immunol (2016) 83(1):33-7. doi:10.1111/sji.12367

32. Gallimore A, Quezada SA, and Roychoudhuri R. Regulatory T Cells in Cancer: where Are We Now? Immunology (2019) 157(3):187-9. doi:10.1111/ imm. 13088

33. Raghavan S, and Quiding-Jarbrink M. Immune Modulation by Regulatory T Cells in Helicobacter Pylori-Associated Diseases. Emiddt (2012) 12(1): 71-85. doi:10.2174/187153012799278974

34. Yang P, Li Q-J, Feng Y, Zhang Y, Markowitz GJ, Ning S, et al. TGF- $\beta$-miR-34aCCL22 Signaling-Induced Treg Cell Recruitment Promotes Venous Metastases of HBV-Positive Hepatocellular Carcinoma. Cancer Cell (2012) 22(3):291-303. doi:10.1016/j.ccr.2012.07.023

35. Baumforth KRN, Birgersdotter A, Reynolds GM, Wei W, Kapatai G, Flavell JR, et al. Expression of the Epstein-Barr Virus-Encoded Epstein-Barr Virus Nuclear Antigen 1 in Hodgkin's Lymphoma Cells Mediates Up-Regulation of CCL20 and the Migration of Regulatory T Cells. Am J Pathol (2008) 173(1): 195-204. doi:10.2353/ajpath.2008.070845

Copyright $\odot 2021$ Zhang, Liu, Yang, Liang, Wang, Wang, Kong, Yuan and Zhou. This is an open-access article distributed under the terms of the Creative Commons Attribution License (CC BY). The use, distribution or reproduction in other forums is permitted, provided the original author(s) and the copyright owner(s) are credited and that the original publication in this journal is cited, in accordance with accepted academic practice. No use, distribution or reproduction is permitted which does not comply with these terms. 